\title{
Wichtige sicherheitsrelevante Information zu Picato ${ }^{\circledR}$ (Ingenolmebutat) Risiko von Hautkrebs bei Patienten mit aktinischer Keratose
}

\author{
Sehr geehrte Frau Doktor, sehr geehrter Herr Doktor
}

In Abstimmung mit Swissmedic möchten wir Sie über den folgenden wichtigen Sicherheitsaspekt informieren:

\section{Zusammenfassung}

- Bei Patienten wurden im Zusammenhang mit einer Behandlung mit Ingenolmebutat Fälle von Plattenepithelkarzinomen gemeldet.

- Einige klinische Studien zeigen eine erhöhte Inzidenz von Hautkrebs.

- Angehörige der Gesundheitsberufe sollten die Patienten darauf hinweisen, auf das Auftreten von Hautveränderungen zu achten und bei solchen Veränderungen sofort ärztlichen Rat einzuholen.

- Ingenolmebutat sollte bei der Behandlung von Patienten mit Hautkrebs in der Anamnese entsprechend mit Vorsicht angewendet werden.

\section{Hintergrund dieser Sicherheitsbedenken}

Picato $^{\circledR}$ (Ingenolmebutat) wird zur topischen Behandlung von nicht-hyperkeratotischen, nicht-hypertrophen aktinischen Keratosen bei Erwachsenen angewendet, wenn die betroffene äussere Hautschicht weder verdickt noch erhaben ist.

In den vorläufigen Ergebnissen der laufenden LangzeitSicherheitsstudie LP0041-63 wird ein Ungleichgewicht bei der Inzidenz von Plattenepithelkarzinomen zwischen dem Ingenolmebutat- und dem Imiquimod-Arm beobachtet. Bei einer Metaanalyse von vier Studien zu dem verwandten Ingenoldesoxat (einer weiteren, nicht zugelassenen Therapie für aktinische Keratosen) wurde beim Vergleich der Wirkstoff- mit den Vehikel-Gruppen nach 14 Monaten ein Anstieg von Hauttumoren festgestellt. Dieser Analyse lag die Inzidenz aller Tumortypen der Haut zugrunde, einschliesslich Basalzellkarzinom, Morbus Bowen und Plattenepithelkarzinom.

Aufgrund der vorliegenden Daten aus der Studie LP004163, der Metaanalyse sowie der Daten aus der LP01051020-Studie zum Vergleich von Ingenolmebutat-Gel mit dem Vehikel (Gel ohne den Wirkstoff) soll die Fachinformation mit den Hinweisen auf ein übermässig erhöhtes Auftreten von Hauttumoren wie Keratoakanthomen, Basalzellkarzinomen, Morbus Bowen und Plattenepithelkarzinomen ergänzt werden, und es soll darauf hingewiesen werden, dass bei der Behandlung von Patienten mit einer Hautkrebserkrankung in der Anamnese Picato ${ }^{\circledR}$ mit Vorsicht anzuwenden ist.

Das Nutzen-Risiko-Verhältnis für Picato ${ }^{\circledR}$ zur Behandlung von Patienten mit aktinischen Keratosen bleibt unverändert.

\section{Empfehlung für Fachpersonen}

Behandelnde Ärztinnen und Ärzte sollten die Patienten darauf hinweisen, auf das Auftreten von Hautveränderungen innerhalb des Behandlungsareals zu achten und bei solchen Veränderungen sofort ärztlichen Rat einzuholen.

\section{International getroffene Massnahmen}

Gemäss der PRAC-Empfehlung werden die Produktinformationen von Picato $^{\circledR}$ in der EU überarbeitet und mit einer Warnung zu den Berichten über Basalzellkarzinome, Morbus Bowen und Plattenepithelkarzinome sowie um den Hinweis, dass Picato ${ }^{\circledR}$ bei der Behandlung von Patienten mit einer Hautkrebserkrankung in der Anamnese mit Vorsicht angewendet werden sollte, ergänzt.

Das Risiko für Hauttumoren sowie die sich daraus ergebenden Implikationen für die Risiko-Nutzen-Bewertung von Picato ${ }^{\circledR}$ werden aktuell weiter durch die EMA überprüft.

\section{Meldung unerwünschter Arzneimittelwirkungen}

Für Meldungen über unerwünschte Arzneimittelwirkungen (UAW) empfiehlt Swissmedic, das dafür entwickelte Meldeportal zu verwenden. Mit dem sogenannten Electronic Vigilance System (ELViS) können UAW gemeldet werden. Alle erforderlichen Informationen sind zu finden unter wWw.swissmedic.ch.

\section{Kontaktangaben}

Wenn Sie Fragen haben oder zusätzliche Informationen benötigen, kontaktieren Sie bitte die medizinische Abteilung von LEO Pharmaceutical Products Sarath Ltd. (Tel. 043 34375 75).

Freundliche Grüsse

Dr. Martina Fähndrich

Medical Advisor

LEO Pharmaceutical Products Sarath Ltd.

Christa Spitznagel

Head of Regulatory Affairs \& Compliance, FvP LEO Pharmaceutical Products Sarath Ltd. 